\title{
Analysis of online plan adaptation for 1.5T magnetic resonance-guided stereotactic body radiotherapy (MRgSBRT) of prostate cancer
}

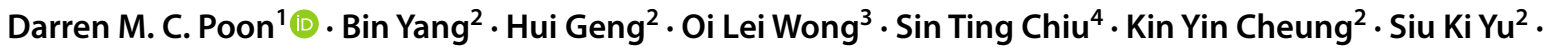 \\ George Chiu ${ }^{4}$ Jing Yuan ${ }^{3}$
}

Received: 10 January 2022 / Accepted: 6 February 2022 / Published online: 24 February 2022

(c) The Author(s), under exclusive licence to Springer-Verlag GmbH Germany, part of Springer Nature 2022

\begin{abstract}
Purpose To analyze and characterize the online plan adaptation of $1.5 \mathrm{~T}$ magnetic resonance-guided stereotactic body radiotherapy (MRgSBRT) of prostate cancer (PC).

Methods PC patients $(n=107)$ who received adaptive 1.5 Tesla MRgSBRT were included. Online plan adaptation was implemented by either the adapt-to-position (ATP) or adapt-to-shape (ATS) methods. Patients were assigned to the ATS group if they underwent $\geq 1$ ATS fraction $(n=51)$; the remainder were assigned to the ATP group $(n=56)$. The online plan adaptation records of $535(107 \times 5)$ fractions were retrospectively reviewed. Rationales for ATS decision-making were determined and analyzed using predefined criteria. Statistics of ATS fractions were summarized. Associations of patient characteristics and clinical factors with ATS utilization were investigated.

Results There were 87 (16.3\%) ATS fractions and 448 ATP fractions (83.7\%). The numbers of ATS adoptions in fractions 1-5 were 29 (29/107, 27.1\%), 18 (16.8\%), 15 (14.0\%), 16 (15.0\%), and $9(8.4 \%)$, respectively, with significant differences in adoption frequency between fractions $(p=0.007)$. Other baseline patient characteristics and clinical factors were not significantly associated with ATS classification (all $p>0.05$ ). Underlying criteria for the determination of ATS implementation comprised anatomical changes ( 77 fractions in 50 patients) and discrete multiple targets ( 15 fractions in 3 patients). No ATS utilization was determined using dosimetric or online quality assurance criteria.

Conclusions This study contributes to facilitating the establishment of a standardized protocol for online MR-guided adaptive radiotherapy in PC.
\end{abstract}

Keywords Prostate cancer (PC) · Magnetic resonance guided radiotherapy (MRgRT) · Stereotactic body radiotherapy $(\mathrm{SBRT}) \cdot$ Online plan adaptation $\cdot$ Adapt-to-shape $(\mathrm{ATS}) \cdot$ Adapt-to-position $(\mathrm{ATP})$

Darren M. C. Poon

darren.mc.poon@hksh.com

1 Comprehensive Oncology Centre, Hong Kong Sanatorium and Hospital, Happy Valley, Hong Kong, Hong Kong SAR, China

2 Medical Physics Department, Hong Kong Sanatorium and Hospital, Happy Valley, Hong Kong, Hong Kong SAR, China

3 Research Department, Hong Kong Sanatorium and Hospital, Happy Valley, Hong Kong, Hong Kong SAR, China

4 Department of Radiotherapy, Hong Kong Sanatorium and Hospital, Happy Valley, Hong Kong, Hong Kong SAR, China

\section{Introduction}

Magnetic resonance guided radiotherapy (MRgRT) represents an innovative and promising new technique for imageguided radiation therapy (IGRT) by taking great advantage of versatile and superior soft-tissue image contrasts of MRI (Lagendijk et al. 2014; Oelfke 2015). With such a highquality image, as a consequence, one of the substantial revolutionary concepts and distinctive features of MRgRT enabled by the hybrid MRI integrated linear accelerator (MR-LINAC) is its capability of daily online treatment plan adaptation based on the on-the-date anatomies of targets and organs-at-risks (OARs) acquired by on-board MRI at each fraction. Daily online plan adaptation promises to further improve tumor control and reduce toxicities compared to the 
existing image-guided adaptive radiotherapy (ART) (Kupelian and Sonke 2014; Hunt et al. 2018; Otazo et al. 2021).

Online MR-guided ART (oMRgART) is defined as the performance of tumor and/or OAR re-contouring and replanning online at the MRgRT treatment fraction, with the patient on the treatment couch (McNair et al. 2020a). The main goals of oMRgART are target coverage improvement and/or OAR sparing (Corradini et al. 2021). Despite the theoretical dosimetric advantage of daily online adaptation, it is recognized that the decision-making process for oMRgART is very complex (McNair et al. 2020a). This process usually involves the synergetic work of a multidisciplinary MRgRT team consisting of the attending radiation oncologist, medical physicists, radiation dosimetrists and/or radiation therapists. Different team members have different roles and responsibilities and jointly influence the final decision regarding oMRgART. Furthermore, many factors, including disease type, patient characteristics, target location and properties, dose constraints and fractionation schemes, to name a few, affect this comprehensive decision-making process (Corradini et al. 2021). More importantly, the decision to deliver oMRgART can greatly increase the complexity of the treatment workflow, prolong the fraction duration, and even increase the probability of patient movement during adaptation, possibly counteracting the dosimetric benefit of oMRgART. (McNair et al. 2020a; Corradini et al. 2021; Kurz et al. 2020; Winkel et al. 2019a; Herk et al. 2018).

In recent years, oMRgART has been rapidly adopted in clinical practice worldwide by institutions that are equipped with either 0.35T (MRIdian system, ViewRay, Inc., Mountain View, CA, USA) or 1.5T (Unity, Elekta, Stockholm, Sweden) MR-LINAC (McNair et al. 2020a). Different vendor-dependent technical solutions and options for plan adaptation are provided on $0.35 \mathrm{~T}$ and 1.5T MR-LINACs. Institutions are recommended to define their specific threshold values and action levels regarding the performance and necessity of oMRgART (Corradini et al. 2021). However, the pattern of oMRgART practice is heterogeneous across different centers, as revealed by the POP-ART RT study and another recent international survey (McNair et al. 2020a; Bertholet et al. 2020). Few studies in the medical literature have reported on institution-specific criteria or protocols for oMRgART decision-making for different types of tumors. To date, no standardized guideline or protocol for the decision-making process for oMRgART has been established in the community of radiation oncology.

Prostate cancer (PC) is one of the major diseases thought to be greatly benefited by oMRgART (Pathmanathan et al. 2018; Cuccia et al. 2021; Tocco et al. 2020). It is also one of the most treated targets by both $0.35 \mathrm{~T}$ and $1.5 \mathrm{~T}$ MRgRT, particularly in the form of ultrahypofractionation, also known as stereotactic body radiotherapy (SBRT) (Ugurluer et al. 2021; Tetar et al. 2021; Bruynzeel et al. 2019; Otterloo et al. 2021).
The recently published European Society for Radiotherapy and Oncology Advisory Committee for Radiation Oncology Practice (ESTRO ACROP) consensus guideline on the use of IMRT for localized PC prefers daily online adaptation for conventionally fractionated radiotherapy and recommends it in case of hypofractionated radiotherapy (Ghadjar et al. 2019). However, how and when to best use oMRgART in clinical practice remains controversial, as revealed in some studies. Alongi et al. reported that they performed oMRgART, namely, adapt-to-shape (ATS) on 1.5T MR-LINAC, for all patients in every fraction of their 1.5T PC MR-guided SBRT (MRgSBRT) (Alongi et al. 2020). Their adoption of oMRgART was also technically supported by a quantitative dosimetric study (Ruggieri et al. 2020). In a more recent 1.5T MRgSBRT study, Poon et al. reported the utilization of ATS in only 29 of 255 (11.4\%) fractions in a cohort of 51 localized PC patients (Poon et al. 2021). Another 0.35T MRgSBRT study reported that manual adjustment on clinical target volume (CTV) was only conducted when necessary to facilitate oMRgART in cases of prostate rotation (Tetar et al. 2021; Bruynzeel et al. 2019). By contrast, a recent clinical study suggested that MRgSBRT without daily adaptation, but with tumor tracking, allowed for successful prostate SBRT in much less time but with equal results in terms of manageable toxicity (Sandoval et al. 2021). Another technical study retrospectively analyzed deformable dose accumulation on scans from four PC patients and found that daily anatomy changes had little impact on the delivered dose, implying an unfavorable role of oMRgART in practice (Yang et al. 2021).

We aimed to analyze and characterize the online plan adaptation used for over 500 treatment fractions of $1.5 \mathrm{~T}$ MRgSBRT of PC in a single center, and investigate correlations between the use of oMRgART and various patient characteristics and clinical factors. We anticipate that these findings will be useful for the community to establish a protocol for optimal online plan adaptation strategy selection in MRgSBRT of PC.

\section{Materials and methods}

\section{Patient selection}

This study was approved by the hospital research committee. The written consent of patients was exempted due to the retrospective nature of the study. All clinically proven PC patients who received irradiation on a $1.5 \mathrm{~T}$ MR-LINAC in our hospital from March 2020 to July 2021 were reviewed. Primary PC with or without intra-pelvic oligometastasis treated with MRgSBRT constituted the majority of these patients. Given the efforts to reduce hospital visits during the COVID-19 outbreak, a proportion of 
patients with post-prostatectomy biochemical recurrence had completed salvage MRgSBRT. PC patients who underwent and successfully finished 5-fraction MRgSBRT, and had a complete clinical record of online plan adaptations for each treatment fraction, were included in the study. Exclusion criteria were previous history of other cancers; treatment with MRgRT in a scheme other than 5-fractionated MRgSBRT, or failure to finish all 5 MRgSBRT fractions; or an incomplete clinical record of online plan adaptations.

\section{MRgRT treatment simulation and planning}

All patients received their computed tomography (CT) and MRI simulation scan on the same day, both in the treatment position with a saline-inflated rectal balloon (QLRAD, Miami, FL, USA). A consistent bladder control protocol was adopted for simulation scans and all MRgRT fractions. MRI simulation images were acquired on a $1.5 \mathrm{~T}$ MRI scanner (Ingenia MR-RT, Philips Healthcare, Best, Netherlands) using a 3D T2-weighted turbo-spin-echo (3D-T2W-TSE) sequence identical to that used for daily online scan on the 1.5T MR-LINAC.

Tissue contouring and image registration were conducted using MIM v.6.9.3 (MIM Software Inc. Cleveland, $\mathrm{OH}, \mathrm{USA}$ ). The institutional target delineation for localized PC has been previously reported (Poon et al. 2021). In general, the CTV of the prostate, seminal vesicles and intra-pelvic oligometastases was contoured by the radiation oncologist on the planning MRI and rigidly registered to the planning CT. For post-prostatectomy MRgSBRT, the CTV consisted of the prostate bed was defined by the Radiation Therapy Oncology Group consensus guidelines (Michalski et al. 2010). The metabolic recurrence as detected in the prostate-specific membrane antigen positron emission tomography (PSMA-PET) scan was registered with the planning MRI/CT and delineated. In patients with a high-risk localized disease with or without the involvement of intra-pelvic nodes (defined by MRI \pm PSMA-PET scan) and post-prostatectomy recurrence, regional pelvic lymphatics (CTV_L) was delineated as defined by the latest NRG consensus (Hall et al. 2021). The planning target volume (PTV) was generated by the isotropic expansion of CTV by $5 \mathrm{~mm}$, except for the $3 \mathrm{~mm}$ in the posterior direction for prostate or prostate $\mathrm{bed} / \mathrm{seminal}$ vesicles. The following OARs were contoured by radiation dosimetrists following institutional contouring guidelines: rectum, bladder, bowel, penis, penile bulb, femoral heads and cauda equina. IMRT plans with a mean of 15 beams were generated using Monaco v.5.40 (Elekta). The details of dose prescription on targets, planning objectives and dose constraints are summarized in Table 1.

\section{Treatment adaptation and delivery}

At each fraction, patients underwent a daily 3D-T2W-TSE scan on the 1.5T MR-LINAC to obtain the on-the-date anatomies to facilitate oMRgART, using either adapt-to-position (ATP) or ATS workflow (Winkel et al. 2019b). Since the use of a rectal balloon and a bladder control protocol helps to reduce intra-/inter-fractional prostate motion, a plan was adopted mainly to compensate for the isocenter shift in many cases. Therefore, the ATP workflow was prioritized over ATS for maximizing treatment efficiency. The decision to choose ATS was jointly made by an attending radiation oncologist, an on-duty medical physicist and an on-duty radiation dosimetrist. In the ATS workflow, the attending oncologist adapted the contours of the CTV, rectum, bladder and bowel through a deformable registration and/or manual adjustment to reflect the on-the-date anatomies. This was followed by the re-optimization of the MRI-based treatment plan. Optimal target coverage was prioritized while restricting the high dose to OARs. After the dosimetric criteria were achieved, an online patient-specific quality assurance (QA) test was performed using an independent monitor unit (MU) calculation approach with RadCalc software (LifeLine Software Inc., Tyler, USA). An additional 3D-T2W-TSE scan followed, for final positional verification. If the patient positional shift was within the tolerance level, dose irradiation was immediately delivered, with or without beam-on motion monitoring using an orthogonal cine MRI.

\section{Data extraction and analysis}

Records of ATP or ATS adaptation for each MRgSBRT fraction were extracted. The attending radiation oncologist, a medical physicist (who attended $>70 \%$ of oMRgART fractions), and a senior radiation dosimetrist (who attended $>70 \%$ of oMRgART fractions) retrospectively reviewed the online MRI images, the reference CT/MRI images and original plans, as well as the ATS adaptive plans, and then analyzed the rationales used for ATS decision-making based on a set of predefined criteria (Table 2). These rationales were originally proposed in an international oMRgART survey (McNair et al. 2020a) then modified based on our institutional protocols and clinical experiences with oMRgART. Note that multiple criteria could account for the ATS decision-making at a single fraction. If disagreement on the ATS rationales occurred among the three reviewers, a consensus was reached after discussion.

We calculated the percentages of ATS and ATP in all fractions, then the frequencies of using ATS at each fraction in the 5-fractionated MRgSBRT treatment course. Statistics of the criteria for ATS decision-making were summarized. The patients were assigned to the ATP group if they had received ATP for all five fractions, and to the ATS group 
Table 1 Planning objectives and dose constraints for organs-atrisk (OARs)

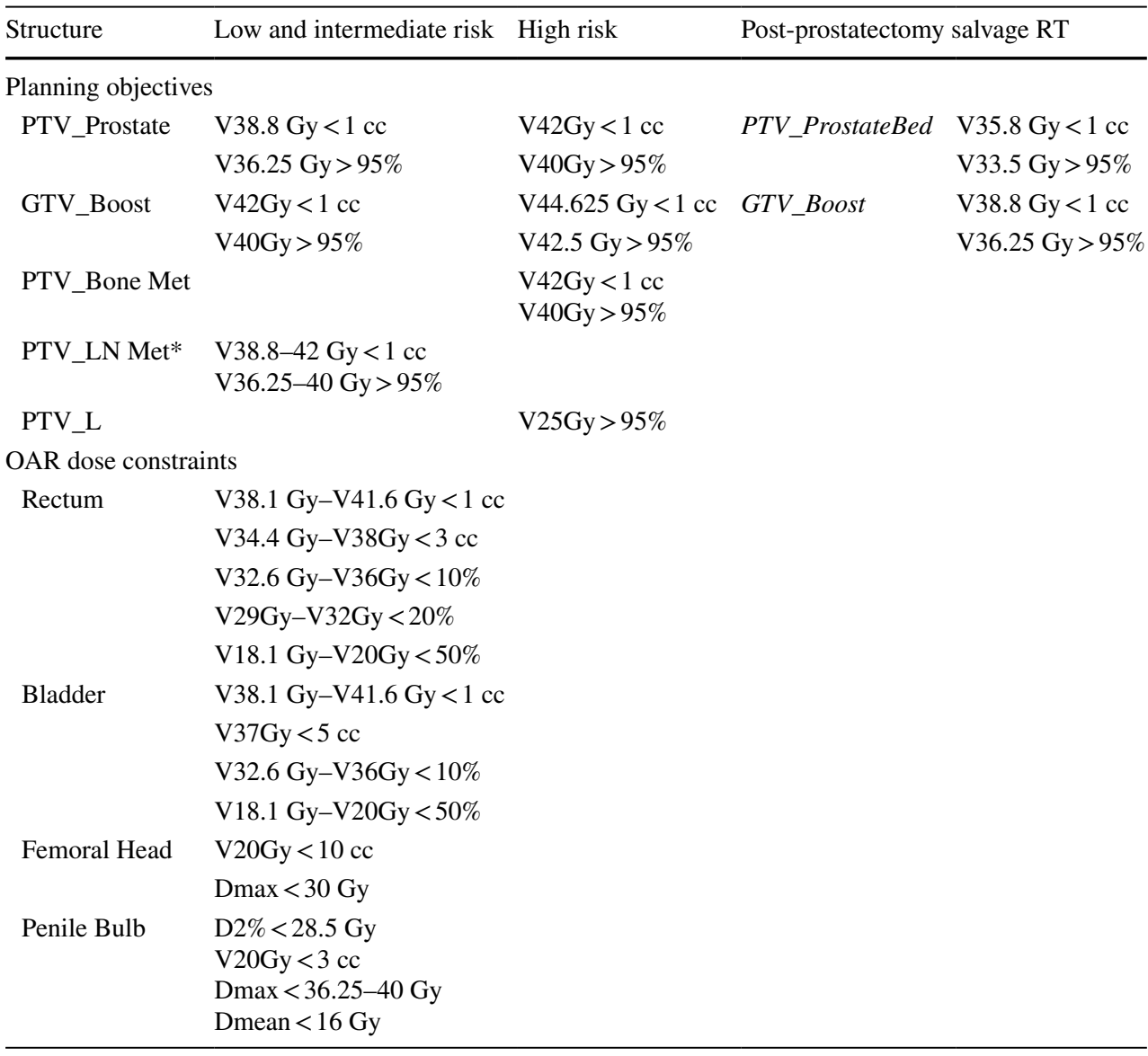

PTV_Prostate planning target volume of whole prostate with $5 \mathrm{~mm}$ ( $3 \mathrm{~mm}$ posteriorly), GTV_Boost gross tumor volume of magnetic resonance imaging (MRI)-visible intra-prostatic lesions with 3-5 mm, PTV Bone Met MRI or prostate-specific membrane antigen (PSMA)-positron emission tomography (PET)defined intra-pelvic bone metastases with 3-5 mm, PTV_LN Met MRI or PSMA-PET defined intra-pelvic lymph node (LN) metastases with 3-5 mm, $P T V \_L$ pelvic lymphatics (CTV_L) defined by the NRG consensus with 5-mm margin

*Final dose prescription depends on the dose constraint of the OARs

Table 2 Predefined criteria to justify the decision for an adapt-to-shape (ATS) plan

Type A Anatomical change criteria

1. Overall gross anatomy changes in target and/or organs at risk

2. Target not covered by planning target volume (PTV)

3. Target too close to edge of PTV ( $2 \mathrm{~mm}$ in all directions)

4. Organs-at-risk close to high-dose area

5. Change of relative position between multiple targets

6. Drastic shape or volume change of body contour

Type B Dosimetric criteria

1. Target dose at that fraction

2. Dose delivered to target in previous fractions

3. Organs-at-risk dose at that fraction

4. Dose delivered to organs-at-risk in previous fractions

Type C Online quality assurance (QA) criteria

1. Online QA of adapt-to-position (ATP) plan fails (mean point dose deviation from the reference plan $>10 \%$ )

Type D Miscellaneous

1. Co-morbidities in patient that may increase toxicity

2. Mandatory ATS workflow for plans with discrete targets to avoid incorrect leaf opening in ATP workflow with Monaco v5.40.00 
if they had received ATS at any MRgRT fraction. Associations between different patient characteristics and clinical factors (including patient age, localized or metastatic PC, number of irradiation targets and the use of rectal spacer) and the ATS/ATP classification were investigated using the odds ratio (OR), Fisher's exact test (for categorized factors), or student's $T$ test (for continuous factors), as appropriate.

All statistical tests were conducted using RStudio 2021.09.0 Build 351 (RStudio PBC, Boston, MA, USA). Descriptive statistics are expressed as means \pm standard deviation. A $p$ value smaller than 0.05 was used to indicate statistical significance.

\section{Results}

Between March 2020 and June 2021, 132 clinically proven PC patients underwent 1.5T MRgRT treatment in our hospital. Among these patients, 25 patients who had undergone conventionally fractionated MRgRT rather than the 5-fraction MRgSBRT were excluded. No patient was excluded based on a history of other cancers, failure to complete the 5 MRgSBRT fractions, or incomplete online adaptation records. Ultimately, 107 consecutive male patients (age $70.1 \pm 8.1$ years, range $46-90$ years) were included in the study. Only one patient had undergone prostatectomy before MRgSBRT.

Of the total $535(107 \times 5)$ fractions, ATS was adopted in $87(16.3 \%)$ and ATP was adopted in 448 (83.7\%) fractions. At fractions $1-5$ of the treatment course, ATS was adopted in $29(29 / 107,27.1 \%), 18$ (16.8\%), 15 (14.0\%), 16 (15.0\%), and $9(8.4 \%)$ fractions, respectively, showing a significant difference in ATS implementation $(p=0.007)$ between fractions (Fig. 1). Of the total 107 patients, $56(52.3 \%)$ received no ATS during their entire treatment (ATP group), while 51 (47.7\%) received at least one ATS fraction (ATS group). The number of patients in the two groups was not significantly different $(p=0.585)$. Among the ATS group, 29 patients received one fraction of ATS $56.9 \%$ of the ATS group; $27.1 \%$ of all patients), 14 received two fractions (27.5\% ATS group; $13.1 \%$ all patients), 5 received three fractions (9.8\% ATS group; $4.7 \%$ all patients), and the remaining 3 received five fractions (5.9\% ATS group; $2.8 \%$ all patients) during their treatment course.

Associations between different patient characteristics and clinical factors and the ATP/ATS group classification are summarized in Table 3. There were insignificant differences between the ATP and ATS groups with regard to patient characteristics and clinical factors (all $p>0.05$ ).

Regarding the decision for ATS implementation in this cohort, criteria under the anatomical changes (Type A) classification were the most frequent, with 77 total fractions among 50 patients. Discrete multiple targets (Type D) was the second most common criterion to trigger the ATS workflow, and accounted for 15 ATS fractions among three patients. Single or multiple criteria were applied for each particular patient, at the discretion of the treatment team, based on the daily MRI images, as exemplified in Fig. 2 (single criterion) and Fig. 3 (multiple criteria). No decisions to implement ATS were based on dosimetric criteria (Type B) or online QA criteria (Type C).

Among all of the Type A (anatomical changes) criteria in our study, "Overall gross anatomy changes in target and/or OARs" (A1) was the most frequent $(n=46)$ criterion for ATS determination, followed by "Target not covered by PTV" (A2) and "OARs close to high-dose area" (A4) (both $n=39$ ), "Drastic shape or volume change of body contour" (A6, $n=22$ ), and "Change of relative
Fig. 1 The number of adapt-toposition (ATP) and adaptto-shape (ATS) adoptions at fraction 1 to fraction 5 during the Magnetic Resonance guided stereotactic body radiotherapy (MRgSBRT) treatment course

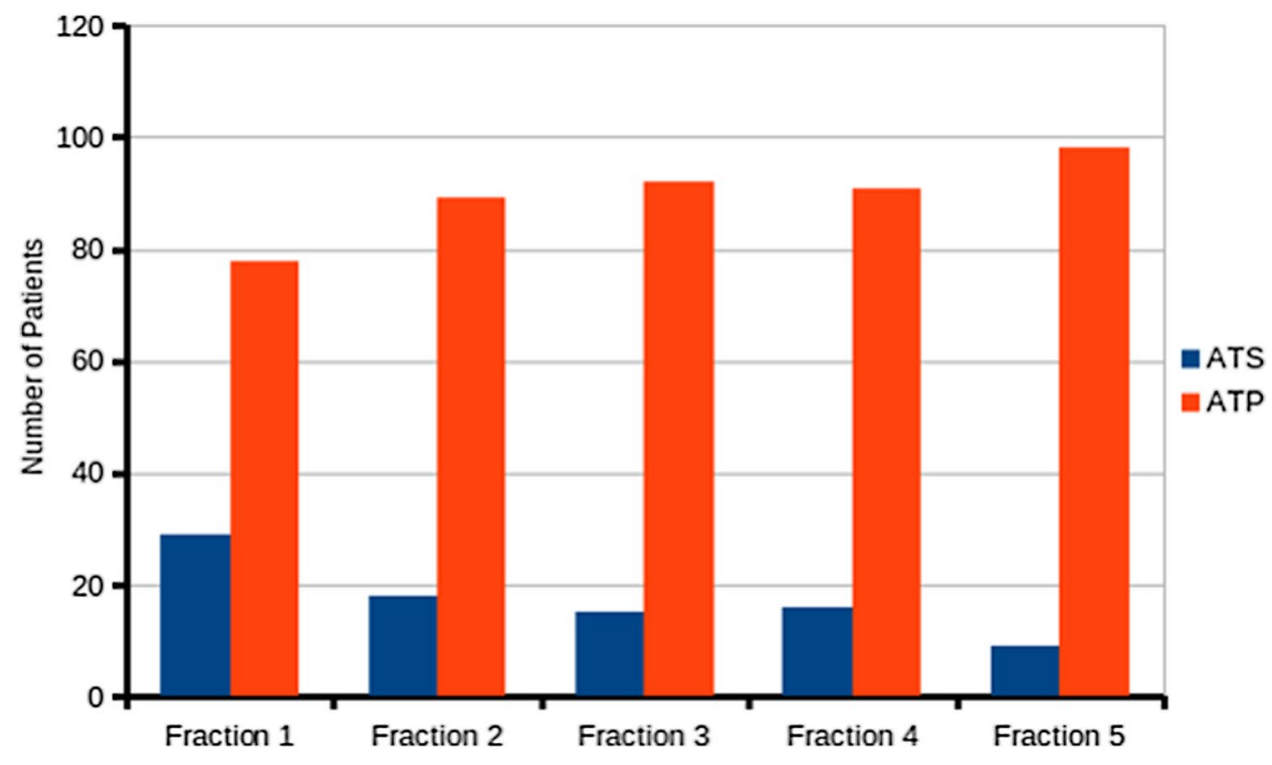


Table 3 Baseline patient characteristics and clinical factors in the ATP and ATS groups

\begin{tabular}{|c|c|c|c|}
\hline Patient characteristic / clinical factor & $\begin{array}{l}\text { ATP group } \\
(N=56)\end{array}$ & $\begin{array}{l}\text { ATS group } \\
(N=51)\end{array}$ & $P$ value (OR) \\
\hline Age (years) & $69.5 \pm 7.8$ & $70.7 \pm 8.4$ & 0.452 \\
\hline Course duration (days) & $14.5 \pm 2.0$ & $14.6 \pm 3.1$ & 0.887 \\
\hline \multicolumn{4}{|c|}{ T stage (localized prostate cancer without nodal or distant metastases) } \\
\hline $\mathrm{T} 1$ & 5 & 4 & \multirow[t]{3}{*}{0.328} \\
\hline $\mathrm{T} 2$ & 31 & 23 & \\
\hline $\mathrm{T} 3$ & 7 & 12 & \\
\hline \multicolumn{4}{|l|}{ Lymph node metastases } \\
\hline No & 44 & 42 & \multirow[t]{2}{*}{$0.448(0.61)$} \\
\hline Yes & 12 & 7 & \\
\hline \multicolumn{4}{|l|}{ Distant metastases } \\
\hline No & 50 & 42 & \multirow[t]{2}{*}{$0.405(1.79)$} \\
\hline Yes & 6 & 9 & \\
\hline \multicolumn{4}{|c|}{ NCCN risk stratification (excluding nodal or distant metastases; $n=25$ ) } \\
\hline Low & 4 & 3 & \multirow[t]{3}{*}{0.147} \\
\hline Intermediate & 27 & 17 & \\
\hline High & 12 & 19 & \\
\hline \multicolumn{4}{|c|}{ Rectal spacer (excluding nodal or distant metastases; $n=25$ ) } \\
\hline No & $n=36$ & $n=30$ & \multirow[t]{2}{*}{$0.578(1.54)$} \\
\hline Yes & $n=7$ & $n=9$ & \\
\hline \multicolumn{4}{|l|}{ Target number } \\
\hline Single target & $n=27$ & $n=25$ & \multirow[t]{2}{*}{$1.000(0.968)$} \\
\hline Multiple targets & $n=25$ & $n=26$ & \\
\hline $\begin{array}{l}\text { CTV (prostate) volume (cc) (excluding mul- } \\
\text { tiple targets) }\end{array}$ & $52.1 \pm 34.4$ & $49.6 \pm 44.3$ & 0.823 \\
\hline
\end{tabular}

NCCN national comprehensive cancer network, CTV clinical target volume, ATP adapt-to-position, ATS adapt-to-shape, $O R$ odds ratio position between multiple targets" (A5, $n=19)$. The least frequent criterion for making the decision to apply ATS was "Target too close to edge of PTV" (A3, n=5). The distribution of each criterion applied for ATS determination at each fraction is illustrated in Fig. 4.

\section{Discussion}

It is anticipated that oMRgART will be used to improve tumor control and/or reduce the toxicity of radiation therapy in PC beyond current IGRT techniques, which are mainly based on CT-based imaging (Kupelian and Sonke 2014; Otazo et al. 2021). However, despite its theoretical advantages, the implementation of online plan adaptation at every fraction is greatly hampered by many factors, which include workflow complexity, unclear criteria for the workflow execution, lack of appropriate precision/fast software, reduced cost efficiency, shortfalls in reimbursement, limited clinical evidence, and more (Bertholet et al. 2020; Glide-Hurst et al. 2021; McNair et al. 2020b). The implementation of oMRgART in clinical practice is far from being well-established or standardized.
Our summary statistics of online plan adaptation for oMRgART fractions conducted on a 1.5T MR-LINAC in a single center revealed that, of a total 535 fractions, the adoption of ATP $(n=448,83.7 \%)$ was far more frequent than that of ATS $(n=87,16.3 \%)$. However, nearly half $(n=51,47.7 \%)$ of the 107 patients received at least one fraction with the implementation of ATS.

In this study, there were no apparent clinical indicators or predictors for ATS adoption. The pattern of ATS utilization was heterogeneous and highly personalized, and greatly dependent on the unpredictable inter-fractional anatomical changes or motions of each individual patient. The only factor that reached statistical significance in the frequency of ATS utilization was the treatment fraction index, with ATS being implemented in $27.1 \%$ and $8.4 \%$ of the 1st and 5th fractions of MRgSBRT, respectively. This might be attributable to the comparatively long interval between the initial simulation scan and the first fraction, which might result in a higher probability of anatomical change than the intervals between subsequent fractions. The decreasing ATS frequency with subsequent fractions might indicate that anatomical changes that occur in response to irradiation might be more substantial at earlier 


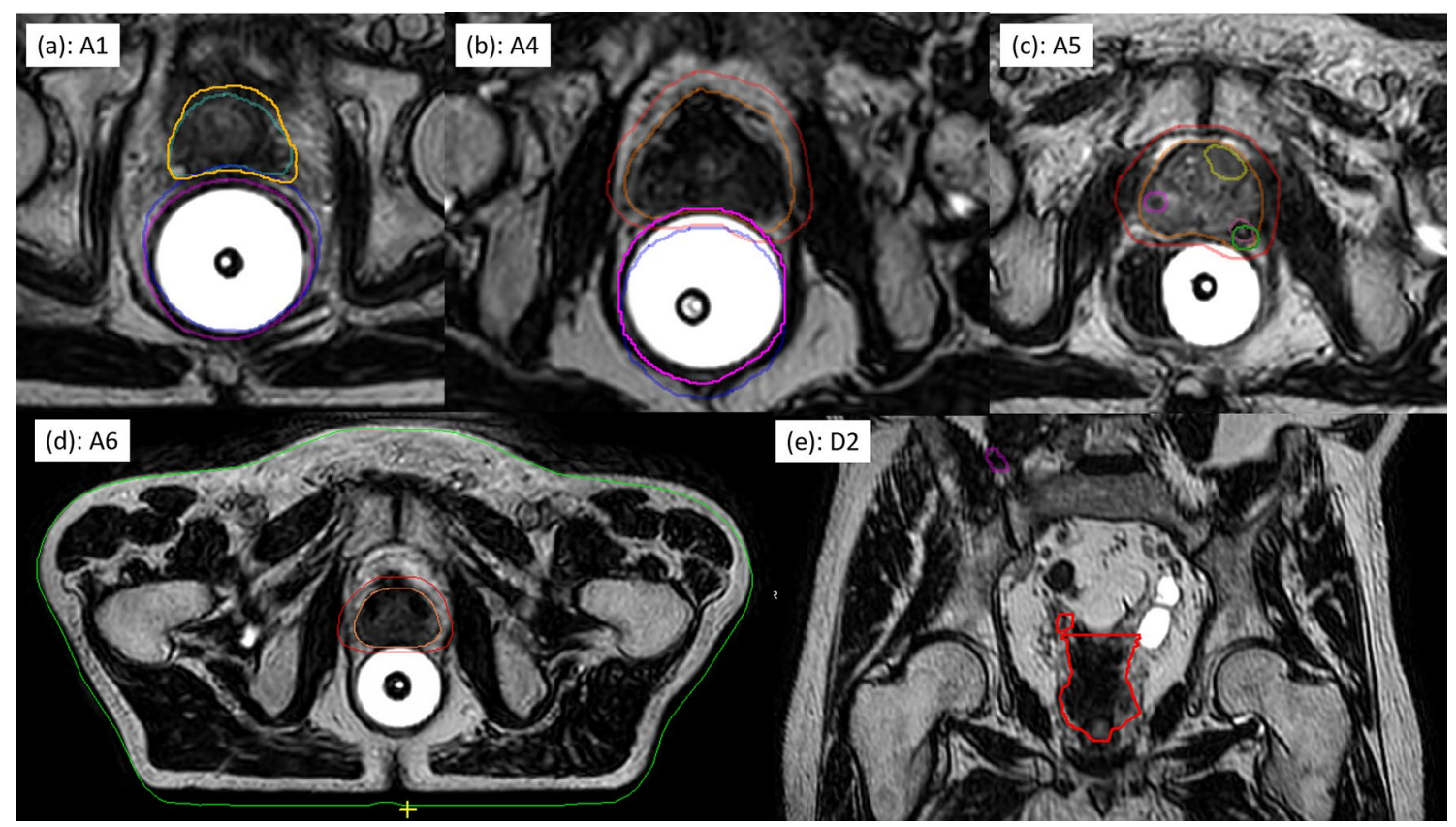

Fig. 2 Examples of daily MRI images when a single criterion was applied for ATS decision-making. (a) Criterion A1: CTV (yellow) and rectum (magenta) in the adaptive plan were modified as compared with the reference CTV (cyan) and rectum (blue); (b) Criterion A4: rectum (magenta) in the adaptive plan was modified due to its proximity to CTV (orange) and PTV (red) as compared with the ref-

fractions than later fractions. However, this postulation should be further verified by a more rigorous quantitative anatomical analysis. Alternatively, the psychological erence rectum (blue); (c) Criterion A5: one boost GTV (pink) in the adaptive plan moved relative to its reference position (green); (d) Criterion A6: body shape changed as compared with the reference body contour (green); (e) Criterion D2: discrete targets of prostate (red) and bone metastasis (magenta)

and physiological stress in patients, which can result in unpredictable anatomical or positional changes, might be expected to diminish gradually with each treatment
Fig. 3 Examples of daily MRI images when multiple criteria were jointly applied for ATS decision-making. (a) Criteria A1+A2: CTV (cyan) in the adaptive plan could not be covered by the reference PTV (red), and its shape changed as compared with the reference CTV (orange); (b) Criteria A1 + A3: CTV (cyan) in the adaptive plan was too close to the reference PTV (red) and its shape changed as compared with the reference CTV (orange); (c) Criteria $\mathrm{A} 1+\mathrm{A} 2+\mathrm{A} 4+\mathrm{A} 5+\mathrm{A} 6$ : CTV (yellow), boost GTV (pink), rectum (magenta) and body contour in the adaptive plan were modified as compared with the reference CTV (cyan), GTV (green), rectum (blue) and body shape (green)

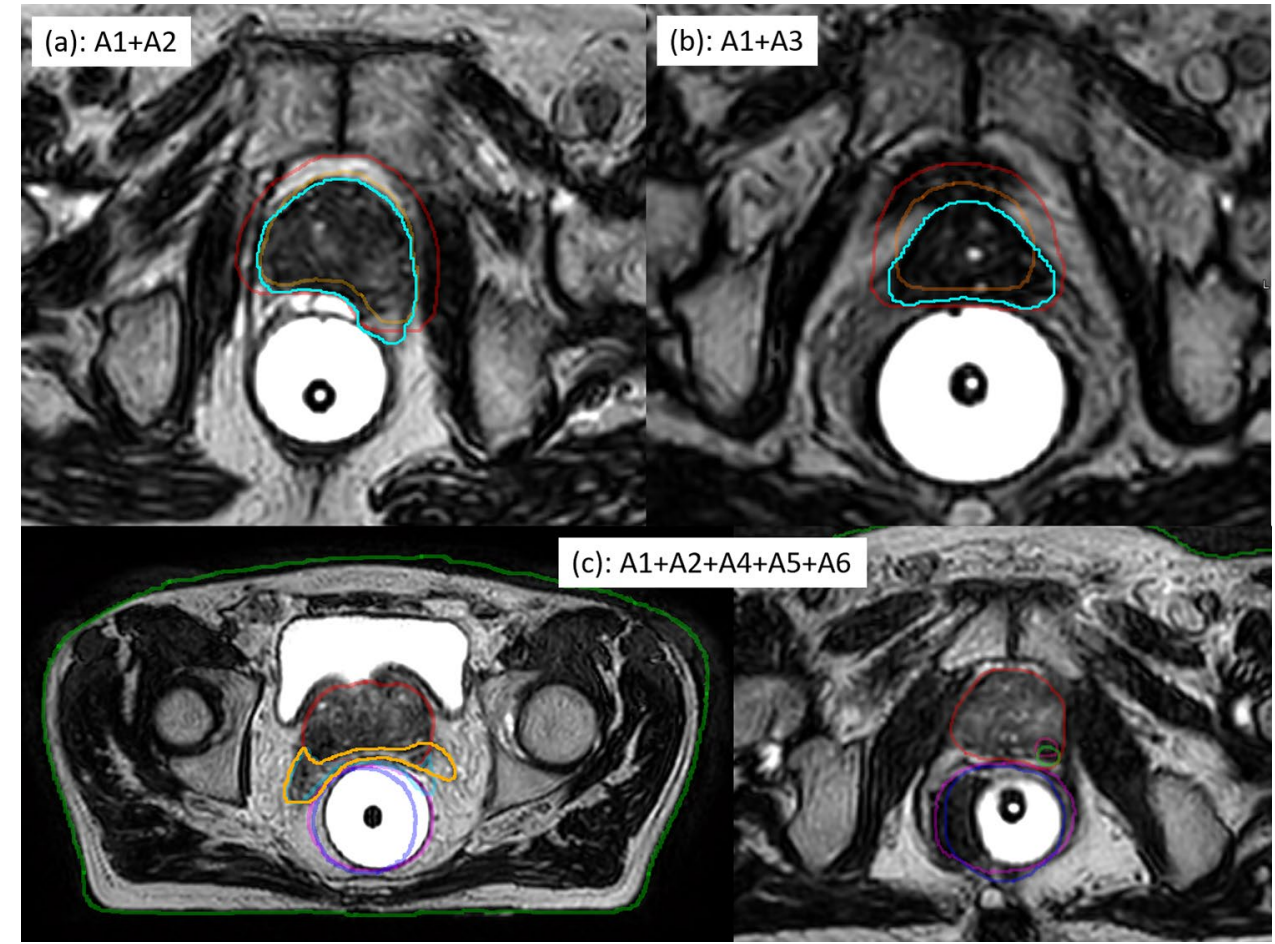


Fig. 4 The distribution of each criterion applied for ATS determination at different fractions during the treatment course

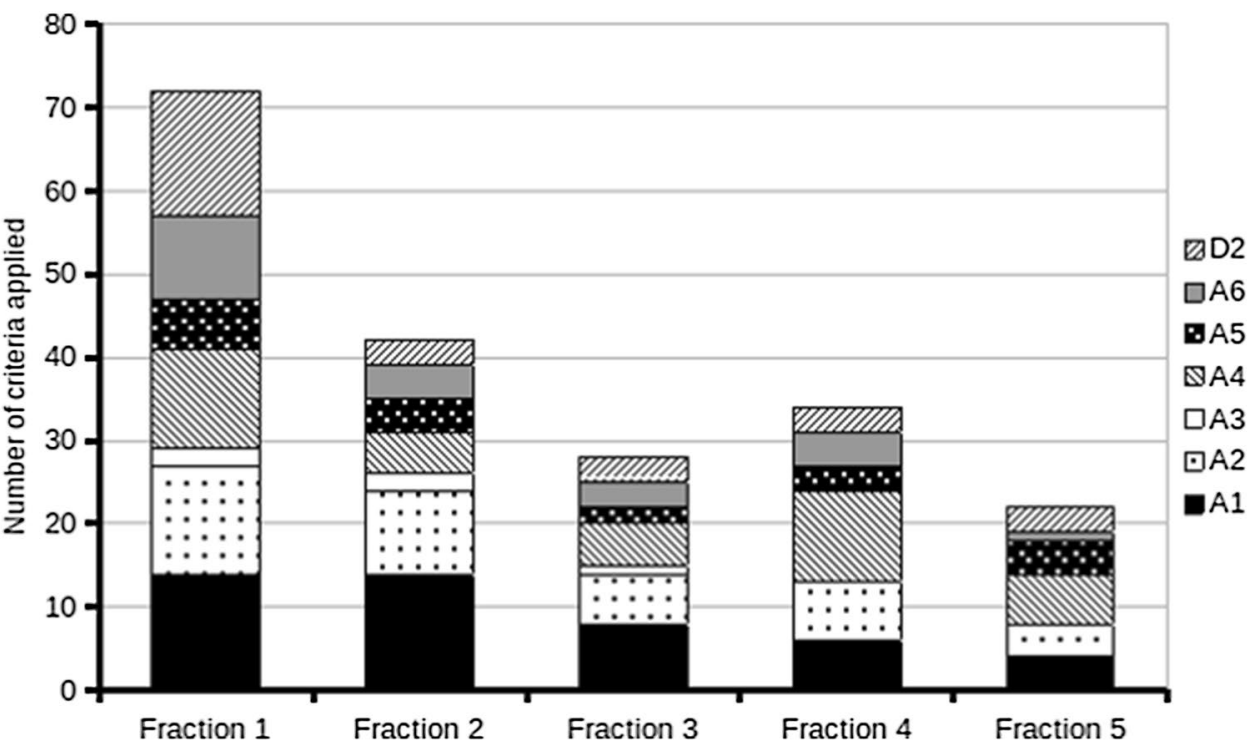

fraction, and could also contribute to the decreasing frequency of ATS during the treatment course.

Since there has been no quantitative, objective or standardized oMRgART protocol established in the community, decision-making on the workflow of online plan adaptation (i.e., ATP vs. ATS) is predominantly dependent on the institutional oMRgART practice guidelines, criteria and tolerance thresholds customized at each center. For instance, the initial daily patient setup on an MR-LINAC might lead to a patient being positioned outside of the institutional tolerance parameter. Whether this situation is handled by patient repositioning or ATS adoption mostly depends on the practice preference at each institution. The patient's bladder and rectum preparation protocol could also heavily determine the anatomical changes and relative positions of these OARs with treatment targets, thus influencing the frequency of ATS adoption. The implementation of ATS due to type A criteria should be highly dependent on the margin setting in the treatment plan (Kim et al. 2019).

The predefined criteria for ATS determination in this study were modified from the criteria formulated in an international survey on oMRgART practice (McNair et al. 2020a). It is worth noting, however, that the ATS decisionmaking in this study did not take into account the importance scores of the original survey criteria. Therefore, the observed frequencies do not necessarily reflect the previous importance rankings of the criteria. Interestingly, the frequency of anatomical change criteria (A1-A4) in this study matched well with their importance score ranking in the original survey. In addition, we found that "Drastic shape/ volume change of body contour" (criterion A6), which was not included in the survey, constituted a much more frequent criterion (22/87 ATS fractions) than "Target too close to edge of PTV" (5/87; criterion A3). Since the registration of target (e.g., prostate) is given a high priority in oMRgART, criterion A3 is infrequently recorded. Metastasis-directed therapy to oligometastases, especially in the form of SBRT, has been shown by various studies (Palma et al. 2019; Decaestecker et al. 2014; Phillips et al. 2020) to be associated with better progression-free survival. Taken together with the significant overall survival benefit of radiotherapy to the prostate primary tumor in patients with low metastatic disease burden (Parker et al. 2018), in this study we chose to include patients with simultaneous SBRT to the prostate primary lesion as well as solitary or multiple intra-pelvic nodal or osseous oligometastases. To accommodate the clinical scenario of multiple-target irradiation in MRgRT, we also introduced an additional criterion of "Change of relative position between multiple targets" (A5) in our practice. This criterion was also frequently adopted (19/87 ATS fractions) in ATS decision-making.

Anatomical changes constituted the predominant type of criteria for ATS decision-making in this study. In sharp contrast, there was no implementation of ATS based on type B "Dosimetric criteria" or type C "Online QA criteria" among the 535 fractions included in this study. One possible reason for the lack of type B criteria for ATS justification is that dosimetric criteria were highly correlated to anatomical change criteria. In many cases, dosimetric criteria are violated by apparent anatomical changes and, as such, they cannot be clearly separated from each other. Meanwhile, there was no clear consensus on the quantitative dose criteria or tolerances to warrant ATS for a single fraction. Furthermore, it would be logistically difficult to perform this time-consuming online quantitative dosimetric assessment beforehand. The absence of type $\mathrm{C}$ criteria can be explained by the well-commissioned independent MU calculation for online patient-specific QA. The mean point dose deviation 
between the online QA tool and Monaco was $-0.2 \% \pm 1.3 \%$, which was far below our tolerance level of $\pm 5 \%$.

Our study has limitations. First, it involved a relatively small sample size of patients with heterogeneous characteristics. Thus, the statistical power in the sub-cohort analysis might not be strong enough. Second, all patients were treated with a 5-fractionated oMRgART scheme, in which the ATS adoption pattern might be substantially different from that in a conventionally fractionated or moderately hypofractionated treatment scheme. Third, the attribution of ATS criteria was conducted retrospectively by three fixed observers, whose procedures for ATS decision-making might not faithfully reflect the actual situation of online adaptation at each fraction. It should also be noted that the ATS decisionmaking procedure is inherently more qualitative and subjective than quantitative and objective. We did not conduct an inter-observer agreement on ATS determination in this study. The predefined criteria modified from the previous survey might not completely cover all possible scenarios for ATS justification, and some criteria might be correlated with each other, as discussed above. Last but not least, there was no quantitative dosimetric analysis or clinical endpoint correlation to justify the true clinical benefit of ATS adoption. This warrants future investigation.

\section{Conclusion}

This study summarized and analyzed the characteristics of online plan adaptation for over 500 1.5T oMRgART fractions in a single center. Our findings provide insight into the patterns and characteristics of online plan adaptation of $1.5 \mathrm{~T} \mathrm{MRgSBRT}$, and may assist with the establishment of a standardized protocol for online MR-guided adaptive radiotherapy of $\mathrm{PC}$.

Acknowledgements The generous donation from Chau Hoi Shuen Foundation in providing financial assistance to some patients in need of MRgRT treatment is acknowledged.

Author contributions All authors contributed to the study conception and design. Material preparation, data collection and analysis were performed by Darren MC Poon, Bing Yang, Hui Geng, Oi Lei Wong and Jing Yuan. The first draft of the manuscript was written by Darren MC Poon and Jing Yuan. All authors commented on previous versions of the manuscript. All authors read and approved the final manuscript.

Funding This study was supported by hospital research project REC-2019-09.

Data availability Due to the nature of this research, participants of this study did not agree for their data to be shared publicly, so supporting data is not available.

\section{Declarations}

Conflict of interest The authors have no relevant financial or non-financial interests to disclose.

Ethical approval This study was approved by the Hong Kong Sanatorium and Hospital Research Ethics Committee REC-2019-09.

Consent to participate Written patient consent form was waived due to the retrospective nature.

Consent for publication The authors give consents for the publication of this manuscript.

\section{References}

Alongi F, Rigo M, Figlia V et al (2020) 1.5 T MR-guided and daily adapted SBRT for prostate cancer: feasibility, preliminary clinical tolerability, quality of life and patient-reported outcomes during treatment. Radiat Oncol 15:69

Bertholet J, Anastasi G, Noble D et al (2020) Patterns of practice for adaptive and real-time radiation therapy (POP-ART RT) part II: offline and online plan adaption for interfractional changes. Radiother Oncol 153:88-96

Bruynzeel AME, Tetar SU, Oei SS et al (2019) A prospective singlearm phase 2 study of stereotactic magnetic resonance guided adaptive radiation therapy for prostate cancer: early toxicity results. Int J Radiat Oncol Biol Phys 105:1086-1094

Corradini S, Alongi F, Andratschke N et al (2021) ESTRO-ACROP recommendations on the clinical implementation of hybrid MR-linac systems in radiation oncology. Radiother Oncol 159:146-154

Cuccia F, Corradini S, Mazzola R et al (2021) MR-guided hypofractionated radiotherapy: current emerging data and promising perspectives for localized prostate cancer. Cancers 13:1791

Decaestecker K, De Meerleer G, Ameye F et al (2014) Surveillance or metastasis-directed Therapy for OligoMetastatic Prostate cancer recurrence (STOMP): study protocol for a randomized phase II trial. BMC Cancer 14:671

Ghadjar P, Fiorino C, Munck Af Rosenschold P, Pinkawa M, Zilli T, van der Heide UA (2019) ESTRO ACROP consensus guideline on the use of image guided radiation therapy for localized prostate cancer. Radiother Oncol 141:5-13

Glide-Hurst CK, Lee P, Yock AD et al (2021) Adaptive radiation therapy (ART) strategies and technical considerations: a state of the ART review from NRG oncology. Int J Radiat Oncol Biol Phys 109:1054-1075

Hall WA, Paulson E, Davis BJ et al (2021) NRG oncology updated international consensus atlas on pelvic lymph node volumes for intact and postoperative prostate cancer. Int J Radiat Oncol Biol Phys 109:174-185

Hunt A, Hansen VN, Oelfke U, Nill S, Hafeez S (2018) Adaptive radiotherapy enabled by MRI guidance. Clin Oncol 30:711-719

Kim JI, Park JM, Choi CH, An HJ, Kim YJ, Kim JH (2019) Retrospective study comparing MR-guided radiation therapy (MRgRT) setup strategies for prostate treatment: repositioning vs. replanning. Radiat Oncol 14:139

Kupelian P, Sonke JJ (2014) Magnetic resonance-guided adaptive radiotherapy: a solution to the future. Semin Radiat Oncol 24:227-232

Kurz C, Buizza G, Landry G et al (2020) Medical physics challenges in clinical MR-guided radiotherapy. Radiat Oncol 15:1-16 
Lagendijk JJ, Raaymakers BW, Van den Berg CA, Moerland MA, Philippens ME, van Vulpen M (2014) MR guidance in radiotherapy. Phys Med Biol 59:R349-369

McNair HA, Wiseman T, Joyce E, Peet B, Huddart RA (2020a) International survey; current practice in on-line adaptive radiotherapy (ART) delivered using magnetic resonance image (MRI) guidance. Tech Innov Patient Support Radiat Oncol 16:1-9

McNair H, Wiseman T, Joyce E, Peet B, Huddart R (2020b) International survey; current practice in on-line adaptive radiotherapy (ART) delivered using Magnetic Resonance Image (MRI) guidance. Tech Innov Patient Support Radiat Oncol 16:1-9

Michalski JM, Lawton C, El Naqa I et al (2010) Development of RTOG consensus guidelines for the definition of the clinical target volume for postoperative conformal radiation therapy for prostate cancer. Int J Radiat Oncol Biol Phys 76:361-368

Oelfke U (2015) Magnetic resonance imaging-guided radiation therapy: technological innovation provides a new vision of radiation oncology practice. Clin Oncol 27:495-497

Otazo R, Lambin P, Pignol JP et al (2021) MRI-guided radiation therapy: an emerging paradigm in adaptive radiation oncology. Radiology 298:248-260

Palma DA, Olson R, Harrow S et al (2019) Stereotactic ablative radiotherapy versus standard of care palliative treatment in patients with oligometastatic cancers (SABR-COMET): a randomised, phase 2, open-label trial. Lancet 393:2051-2058

Parker CC, James ND, Brawley CD et al (2018) Radiotherapy to the primary tumour for newly diagnosed, metastatic prostate cancer (STAMPEDE): a randomised controlled phase 3 trial. Lancet 392:2353-2366

Pathmanathan AU, van As NJ, Kerkmeijer LGW et al (2018) Magnetic resonance imaging-guided adaptive radiation therapy: a "game changer" for prostate treatment? Int J Radiat Oncol Biol Phys 100:361-373

Phillips R, Shi WY, Deek M et al (2020) Outcomes of observation vs stereotactic ablative radiation for oligometastatic prostate cancer: the ORIOLE Phase 2 Randomized Clinical Trial. JAMA Oncol 6:650-659

Poon DMC, Yuan J, Wong OL et al (2021) 1.5T magnetic resonanceguided stereotactic body radiotherapy for localized prostate cancer: preliminary clinical results of clinician- and patient-reported outcomes. Cancers 13:628
Ruggieri R, Rigo M, Naccarato S et al (2020) Adaptive SBRT by $1.5 \mathrm{~T}$ MR-linac for prostate cancer: on the accuracy of dose delivery in view of the prolonged session time. Phys Med 80:34-41

Sandoval ML, Youssef I, Latifi K et al (2021) Non-adaptive MR-guided radiotherapy for prostate SBRT: less time, equal results. J Clin Med 10:3396

Tetar SU, Bruynzeel AME, Oei SS et al (2021) Magnetic resonanceguided stereotactic radiotherapy for localized prostate cancer: final results on patient-reported outcomes of a Prospective Phase 2 Study. Eur Urol Oncol 4:628-634

Tocco BR, Kishan AU, Ma TM, Kerkmeijer LGW, Tree AC (2020) MR-guided radiotherapy for prostate cancer. Front Oncol 10:616291

Ugurluer G, Atalar B, Zoto Mustafayev T et al (2021) Magnetic resonance image-guided adaptive stereotactic body radiotherapy for prostate cancer: preliminary results of outcome and toxicity. $\mathrm{Br} \mathrm{J}$ Radiol 94:20200696

van Herk M, McWilliam A, Dubec M, Faivre-Finn C, Choudhury A (2018) Magnetic resonance imaging-guided radiation therapy: a short strengths, weaknesses, opportunities, and threats analysis. Int J Radiat Oncol Biol Phys 101:1057-1060

van Otterloo SRM, Christodouleas JP, Blezer ELA et al (2021) Patterns of care, tolerability and safety of the first cohort of patients treated on a novel high-field MR-Linac within the MOMENTUM Study: Initial results from a prospective Multi-Institutional Registry. Int J Radiat Oncol Biol Phys. https://doi.org/10.1016/j.ijrobp.2021. 07.003

Winkel D, Bol GH, Werensteijn-Honingh AM et al (2019a) Evaluation of plan adaptation strategies for stereotactic radiotherapy of lymph node oligometastases using online magnetic resonance image guidance. Phys Imaging Radiat Oncol 9:58-64

Winkel D, Bol GH, Kroon PS et al (2019b) Adaptive radiotherapy: the Elekta unity MR-linac concept. Clin Transl Radiat Oncol 18:54-59

Yang J, Vedam S, Lee B et al (2021) Online adaptive planning for prostate stereotactic body radiotherapy using a 1.5 Tesla magnetic resonance imaging-guided linear accelerator. Phys Imaging Radiat Oncol 17:20-24

Publisher's Note Springer Nature remains neutral with regard to jurisdictional claims in published maps and institutional affiliations. 